\title{
On an Entropy-based Performance Analysis in Sports
}

\section{Micael S. Couceiro ${ }^{1,2,3 *}$, Filipe M. Clemente ${ }^{3,4}$, Gonçalo Dias ${ }^{3,4}$, Pedro Mendes ${ }^{3}$, Fernando M. L. Martins $^{3,5}$ and Rui S. Mendes ${ }^{3}$}

1 Institute of Systems and Robotics, University of Coimbra, Pinhal de Marrocos, Polo II, 3030-290, Coimbra, Portugal; E-Mail: micaelcouceiro@isr.uc.pt (MSC)

2 Ingeniarius, Lda., Rua da Vacariça, n.37, 3050-381, Mealhada, Portugal; E-Mail: micael@ingeniarius.pt (MSC)

3 Polytechnic Institute of Coimbra, ESEC, RoboCorp, ASSERT, Rua Dom João III Solum, 3030-329 Coimbra, Portugal; E-Mail: micael@isec.pt (MSC); filipe.clemente5@gmail.com (FC); goncalodias@fcdef.uc.pt (GD); $\quad$ pmendes@esec.pt (PM); fmlmartins@esec.pt (FM); rmendes@esec.pt (RM)

4 Faculty of Sport Sciences and Physical Education, University of Coimbra, Estádio Universitário de Coimbra, Pavilhão 3, 3040-156 Coimbra, Portugal; E-Mail: filipe.clemente5@gmail.com (FC); goncalodias@fcdef.uc.pt (GD)

5 Instituto de Telecomunicações, Delegação da Covilhã, Convento Santo António, 6201-001 Covilhã, Portugal; E-Mail: fmlmartins@esec.pt (FM)

* Author to whom correspondence should be addressed; E-Mail: micaelcouceiro@isr.uc.pt Tel.: +351961171409.

Received: 17 September 2014 / Accepted: 30 September 2014 / Published: 3 November 2014

\begin{abstract}
This paper discusses the major assumptions of influential ecological approaches on the human movement variability in sports and how it can be analyzed by benefiting from well-known measures of entropy. These measures are exploited so as to further understand the performance of athletes from a dynamical and chaotic perspective. Based on the presented evidences, entropy-based techniques will be considered to measure, analyze and evaluate the human performance variability under three different case studies: $i$ ) golf; $i$ ) tennis; and iii) soccer. At a first stage, the athletes' performance will be analyzed at the individual level by considering the golf putting (pendulum movement) and the tennis serve (ballistic movement). Under these gestures, the approximate entropy is considered to extract the variability inherent to the process variables. Afterwards, the athletes' performance will be analyzed at the collective level by considering the soccer case (team sport). To that end,
\end{abstract}


both approximate entropy and Shannon's entropy are mutually considered to assess the variability of football players' trajectory. To outline the applicability of entropy-based measures to analyze sports, this article ends with an overall reflection about the potential of such measures towards an increased understanding on the overall human performance. This methodology proves to be useful to provide decisive information and feedback for coaches, sports analysts and even for the athletes.

Keywords: sport sciences; chaos and nonlinear dynamics; entropy; performance analysis.

PACS Codes: 37Fxx; 37Mxx; 01.80.+b; 05.45.Tp

\section{Introduction}

Humans can perform an incredibly wide range of movements [1]. In all of those movements, even in pendulum and very regular ones, such as the golf putting, the variability is always inherent to human actions [2]. One of the most understandable reasons behind such variability is the large number of degrees of freedom from human's motor system [3]. These degrees of freedom are associated to the three groups of human joints (immovable joint, amphiarthros is joint and diarthrosis/synovial joints) which accounts for a large range of motion combinations [4]. Despite human variability, it is well known that there is an invariant part of each specific action which can be observable from repetition to repetition [5]. The more the invariant part of a specific action is, the more it can be deemed as stable. Nevertheless, even the most stable movements, such as standing still in the same place, inevitably have small oscillations inherent to the muscles contractions and joints protection cycles [6]. Therefore, one can state that the variability represents the complexity of a given movement, or it may simply represent an early stage of motor learning or the presence of task constraints [7]. In other words, a lower level of variability arises from a more adaptive organization over the individual degrees of freedom, while, in contrast, a higher level of variability is related to a lower dimensional output from individual effectors [5]. These kinds of evidences are imperative in many fields of human interaction, such as medicine, physiotherapy and sports. In the latter, the ability an athlete has in performing a stable action even under complex scenarios is challenging and, in spite of that, training is necessary [2].

To understand how variable or unstable a given athlete is, most of the proposed approaches presented in the literature have been focused in studying the product variables (e.g., number of goals, points or any other final outcome). In these approaches, the variability is usually expressed as a standard statistical measure, such as the standard deviation associated with the final outcome [5]. Nevertheless, the variability of an athlete must be understood and perceived during the execution of the task, i.e., within the process variables (e.g., angular positioning of joints, acceleration or muscles contraction). Process variables are key elements that may help coaches in identifying the appropriate constraints associated to a given athlete and increase the athlete performance. Bearing this idea in mind, next section overviews the current state-of-the-art, discussing how variability has been used and understood in sport sciences as a measure of the human performance. 


\subsection{Related Work}

Many researchers interpret variability in sport sciences as a mechanism to force athletes into adapting and stabilizing their actions to constraints (e.g., individual, task and environment) [8]. The variability then contributes to an adaptive human motor control and may be adjusted to produce a desirable outcome. For instance, in most sport modalities, it is noticeable that athletes' morphologic (e.g., weight and height) and functional (e.g., motivation and fatigue) characteristics may affect their performance. These characteristics are intrinsic to the inter-individual profiles that distinguish athletes during the process of motor execution [9].

Human movement variability can be defined as the typical variations that are inherent to the motor performance and which may be observed across multiple repetitions of a given task [10], [11]. So, we may easily perceive that an athlete has to adapt to the multiple conditions of variability. Such adaptation involves a variety of strategies generated to solve individual motor problems. As an example, the studies of [8], [9], [19] claimed that a golfer faces several constraints, being susceptible to a high variability of practice conditions that require systematic adaptation mechanisms. The athlete is then faced with multiple possible ball trajectories (e.g., either linear or curvilinear), slopes (e.g., either ascending or descending), adverse weather conditions (e.g., sun, rain, wind and snow) and different greens (e.g., short grass, high grass, ill-treated grass, grass with holes and sand, among others). Despite these claims, information concerning a specific way that an athlete is able to adapt his, or her, process to a given context and how variability conditions affect that is scarce. Henceforth, one may consider it as decisive to understand how variability can be perceived within sport movements.

The study of [9] concluded that the problem of individuality in sport movements is not confined in ideal or standardized techniques, but withholds a variety of strategies that may be employed according to the specificity of each athlete. In other words, nonlinear techniques, like entropy-based measures, provide quantitative and qualitative information about a certain motor system tendency by tracking human movement patterns [12]. Unlike cognitive theories that support traditional motor control models, e.g., [13] and [13] considers variability to be a negative factor in learning, the nonlinear perspective supports that "embedding chaos" into the process may be necessary to establish new human movement patterns [15]. However, only some few studies, such as [9], [6] and [17], analyzed sports from a nonlinear perspective, using tools like entropy. This recent use of nonlinear measures in sports have fostered the evaluation of not only the magnitude of the human variability, but also its temporal structure, thus allowing to describe complex conditions that cannot be described using only linear tools [12]. In fact, according [12], traditional linear tools may even "conceal" the true structure of motor variability.

In spite of this, nonlinear techniques, like Shannon's Entropy and the Approximate Entropy, are extremely useful to further analyze the human movement within sports context. These measures aim for the evaluation of the overall temporal variability structure, quantifying and qualifying how a set of values in a particular distribution are organized in time or even across a range of time scales [11], [12]. These advances shall open new research topics under the training and tactical scope, thus narrowing the gap between sports sciences and sports coaching [16]. 


\subsection{Statement of Contribution}

This paper starts by presenting a brief overview on entropy-based measures, focusing on the applicability of Shannon's entropy and the approximate entropy within sports context (Section 2). This is further exploited in Section 3, wherein these measures are considered to study the motor control variability in three completely different modalities, namely the golf putting, the tennis serve and the football. The variability emerging from the athletes' performance is considered as a measure to compare their performance under different practice conditions (intrapersonal analysis) and between different athletes (interpersonal analysis). The paper ends with conclusions and final remarks (Section 4).

\section{Entropy}

The human movement variability can be described as motor performance variations over multiple repetitions for the same task [5]. Despite similarities between repetitions, the multiple constraints inherent to the environment, the task and even the athlete's body, lead to inevitable differences, thus resulting in a certain degree of variability [3]. From an evaluation perspective, the human movement oscillations can be evaluated, as any other time-series, by benefiting from entropy-based techniques [12], which allow to identify the variability in a spatio-temporal perspective. Despite the multiple entropybased techniques one may consider to measure sports variability, both Shannon's entropy [18] and approximate entropy (ApEn) [24] have been the ones most widely used. Hence, let us briefly describe both methods.

\subsection{Shannon's entropy}

The entropy measure proposed by Shannon was initially considered to quantify the expected value of the information contained in a message, which can be measured in units such as bits [18]. However, the applicability of Shannon's entropy is wide; from the study of birds' vocal repertories [19] to the heart rate variability [20]. Within sports context, some studies have benefitted from Shannon's entropy to measure the athletes' variability, mainly applying it to their trajectory [18]. In this specific situation, Shannon's entropy is applied as in any other images processing problem, by considering the relevant information about the spatial variability of athletes. To apply Shannon's entropy to a generic image, one should consider the histogram entry of intensity value $i, h_{i}$, to first retrieve the probability mass function as [22]:

$$
p_{i}=\frac{h_{i}}{N_{c}}
$$

wherein $N_{c}$ is the total number of cells (i.e., the spatial resolution of a field). Shannon's entropy can then be calculated as [18], [23]: 


$$
E=-\sum_{i} p_{i} \log _{2} p_{i}
$$

Considering a soccer field of $N_{c}$ cells, equation (2) returns the values of entropy defining the variability of a given athlete's trajectory based on the time a he stands on a cell. High values represent a large variability, which may as well describe a given chaotic or even completely random trajectory spread all over the field. On the other hand, low values represent a small human movement variability, which may as well mean that the athlete presents a rather periodic or even completely steady trajectory (e.g., have an homogenous trajectory all over the field or being in the same position).

Shannon's entropy quantifies the information expected value associated to a discrete random variable [23]. As such, Shannon's entropy can also be used as a measure of uncertainty, or unpredictability. For instance, for a uniform discrete distribution, i.e., when all the values of the distribution have the same probability, Shannon's entropy reaches its maximum. The minimum value of Shannon's entropy then corresponds to perfect predictability, while higher values of Shannon's entropy are related to a lower degree of predictability [23]. As it considers the variability over time, the entropy can be seen as a more general measure of uncertainty when compared to the variance or the standard deviation [23]. Another interesting feature is that, whilst both entropy and variance reflect the degree of concentration for a particular distribution, they are rather different: while the variance measures the concentration around the mean, the entropy measures the diffuseness of the density irrespective of the location parameter.

\subsection{Approximate Entropy}

The approximate entropy $(A p E n)$ was first introduced by Pincus as a measure for characterizing the regularity in relatively short and potentially noisy data [24]. More specifically, ApEn quantifies the degree of irregularity or randomness within a time-series (of length $N$ ) and has already been widely applied to (non-stationary) biological systems to dynamically monitor the system's "health" [25]. The conceptual idea is rooted in the work of Grassberger and Procaccia [26] and makes use of the distances between sequences of successive observations. ApEn then analyses the time-series looking for similar epochs, in which more similar and more frequent epochs lead to lower values of $A p E n$ [25].

From a qualitative point- of- view, given $N$ points, $A p E n$ is approximately equal to the negative logarithm of the conditional probability that when two sequences similar for $m$ points remain similar within a tolerance $r$ at the next point [25]. Smaller ApEn values indicate a greater chance that a set of data will be followed by similar data (regularity) [26], i.e., greater regularity. Contrariwise, larger $A p E n$ values points toward a lower chance of repeatable data (irregularity), i.e., more disorder, randomness and 
system complexity. In other words, a low/high $A p E n$ value reflects a high/low degree of regularity. It is also noteworthy that $A p E n$ detects changes in underlying episodic behavior not reflected in peak occurrences or amplitudes.

The techniques for estimating the approximate entropy can be considered as a process represented by a time-series and related statistics [24]. Let us consider that the whole data of $t$ samples (i.e., seconds) is represented by a time-series as $u(1), u(2), \ldots, u(\mathrm{~N})$, from measurements equally spread out in time [17]. These samples form a sequence of vectors $x(1), x(2), \ldots, x(N-m+1) \in \mathbb{R}^{1 \times m}$, each one defined by the array $x(i)=\left[\begin{array}{llll}u(i) & u(i+1) & \cdots & u(i+m-1)\end{array}\right] \in \mathbb{R}^{1 \times m}$. Parameters $N_{t}, m$, and $\varepsilon$ must be stationary for each calculation. The parameter $N$ represents the length of the time-series (i.e., number of data points of the whole series), $m$ denotes the length of sequences to be compared and $\varepsilon$ is the tolerance for accepting matches. So, one can define [17]:

$$
C_{i}^{m}(\varepsilon)=\frac{\text { numberof } x(j) \operatorname{suchthatd}(x(i), x(j)) \leq \varepsilon}{N-m+1} .
$$

for $1 \leq i \leq N-m+1$. Based on Takens' work(11), one can defined the distance $d(x(i), x(j))$ for vectors $x(i)$ and $x(j)$ as:

$$
d(x(i), x(j))=\max _{k=1,2, \ldots, m}|u(i+k-1)-u(j+k-1)|
$$

From the $C_{i}^{m}(\varepsilon)$, it is possible to define (11):

$$
C_{i}^{m}(\varepsilon)=\left(N_{c}-m+1\right)^{-1} \sum_{i=1}^{N_{c}-m+1} C_{i}^{m}(\varepsilon)
$$

and the correlation dimension as:

$$
\eta_{m}=\lim _{\varepsilon \rightarrow 0} \frac{\ln \left(C^{m}(\varepsilon)\right)}{\ln \varepsilon}
$$

for a sufficiently large $m$. The limit in [6] exists for many chaotic attractors and this procedure is frequently applied to experimental data. In fact, researchers seek a "scaling range" of $\varepsilon$ values for which $\frac{\ln \left(C^{m}(\varepsilon)\right)}{\ln \varepsilon}$ is nearly constant for large $m$, and they infer that this ratio is the correlation dimension. In some studies, it was concluded that this procedure establishes deterministic chaos [17]. 
Let us define the following relation:

$$
\Phi^{m}(\varepsilon)=\left(N_{c}-m+1\right)^{-1} \sum_{i=1}^{N_{c}-m+1} \ln C_{i}^{m}(\varepsilon) .
$$

One can define the approximate entropy as:

$$
A p E n=\Phi^{m}(\varepsilon)-\Phi^{m+1}(\varepsilon)
$$

A preliminary conclusion suggests that choice of $\varepsilon$ of the standard deviation of the data ranging from 0.1 to 0.2 would produce reasonable statistical validity of $A p E n$ [17].

For that range of values, it is possible to classify a given time-series using the approximate entropy ApEn as [20]: i) periodic ( 0); ii) chaotic (0.1 until 1.4); and iii) random (> 1.5).In summary, the presence of repetitive patterns of fluctuation in a time-series (periodic time-series) renders it more predictable than a time-series in which such patterns are absent (chaotic or random time-series).

\section{Case Studies}

This section explores the applicability of the entropy measures presented in the previous section by considering three case studies: $i$ ) the golf putting; $i$ ) the tennis serve; and iii) the football.

\subsection{Golf}

To describe the golf putting variability, it has been established that nonlinear techniques, such as the approximate entropy, should allow unraveling the structure of a mathematical representation of this movement. Most traditional research around the putting has been trying to quantify the motor performance of athletes through the mean, standard deviation and coefficient of variation, which take into consideration the individual characteristics of athletes and are mostly based upon statistical effects to characterize the learning and training of this gesture (see [8, 9 and 10]). However, as a human movement, even as "simple" as the golf putting, is seen as a nonlinear system capable of producing solutions to solve motor problems, it requires special attention and in-depth research. In fact, each golfer has different morphological and functional characteristics that may represent a determined performance profile, typically known as "signature" or "digital fingerprint".

The way athletes adapt to the variability that emerges from the putting execution and how they selforganize their performance toward the task constraints was then investigated. The sample consisted of 10 adult male golfers (33.80 \pm 11.89 years), right-handed and experts (10.82 \pm 5.40 handicap), including the European champion of pitch and putting (season 2012/2013). The athletes executed the task on a rectangular green carpet, replicating a fast putting surface able to provide a ball speed up to $10 \mathrm{~m} / \mathrm{s}$ (Figure 1a). 
The procedure comprised on three studies. In the first study (St1), golfers had to execute the putting at $1(\mathbf{1 m}), 2(\mathbf{2 m}), 3(\mathbf{3 m})$ and $4(\mathbf{4 m})$ meters away from the hole, without any additional constraint. In the second study (St2), a slope was placed between the hole and the 2 meters distance. In that study, golfers had to execute the putting at 2 (2m), 3 (3m) and 4 (4m) meters away from the hole. Finally, in the third study (St3) an additional constraint was added by obliging golfers to execute the putting on the left and right side of the carpet at $25^{\circ}$ in relation to the hole (see Angle One (A1) and Angle Two (A2) in Figure 1a).

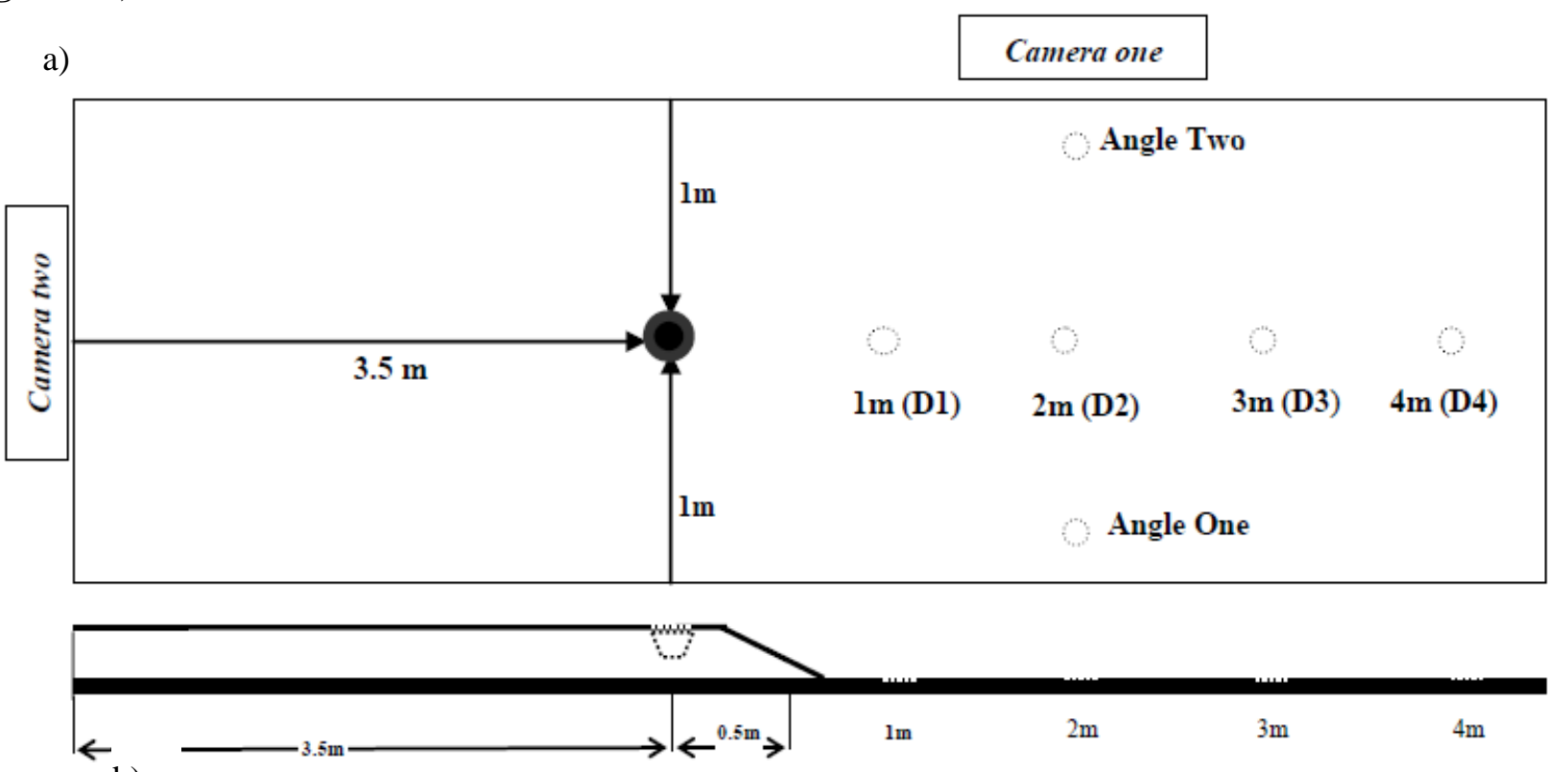

b)

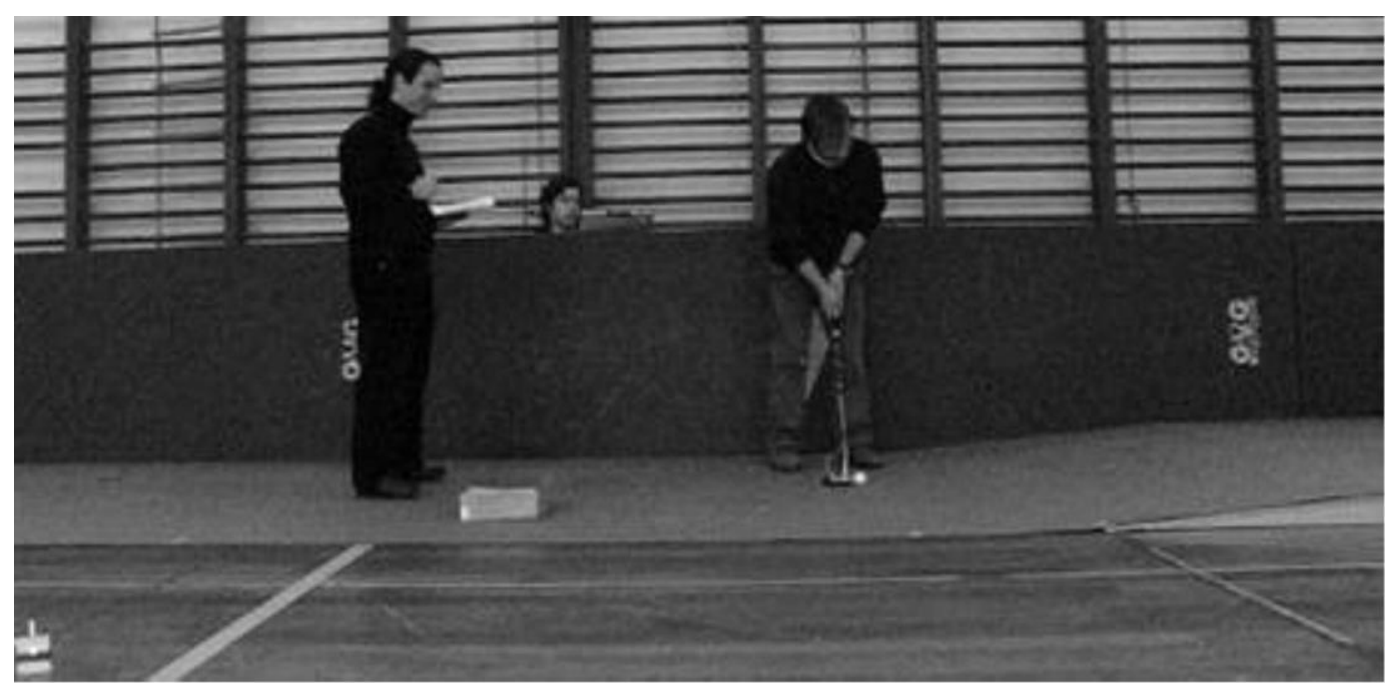

Figure 1.a) Top and side views of the experimental apparatus; b) captured frame with the experimental setup prepared for the second study (St2) 2 meters (2m) away from the hole (adapted from [8] and [9]).

The trajectory of the putter was acquired using auto tracking methodologies comprising on detection and estimation technique as presented in our previous work [8]. To that end, a camera at $210 \mathrm{~Hz}$ was placed in front of golfers so as acquire the most relevant process variables, namely the horizontal trajectory of the putter (Figure 1b). This pre-processing step would then return the trajectory of the each putting trial. Nevertheless, in order to evaluate the putting over the whole procedure, one needs to 
generate a single time-series that may represent the overall putting data over time in all the trials under that same condition. In other words, after obtaining the data of each putting one wishes to evaluate as a hole, it is necessary to concatenate the necessary data. For instance, Figure $\mathbf{2}$ depicts 30 trials of a single golfer under a specific practice condition concatenated into a single time-series. Generally speaking, the function from Figure 2 represents the time series characteristic of a golfer's movement in a one practice condition. In the case represented in Figure 2, one can confirm that, although the gesture is mostly periodic, the golfer still presents some variability at the level of putting execution (the amplitude and duration of the movement slightly diverges throughout the trials).

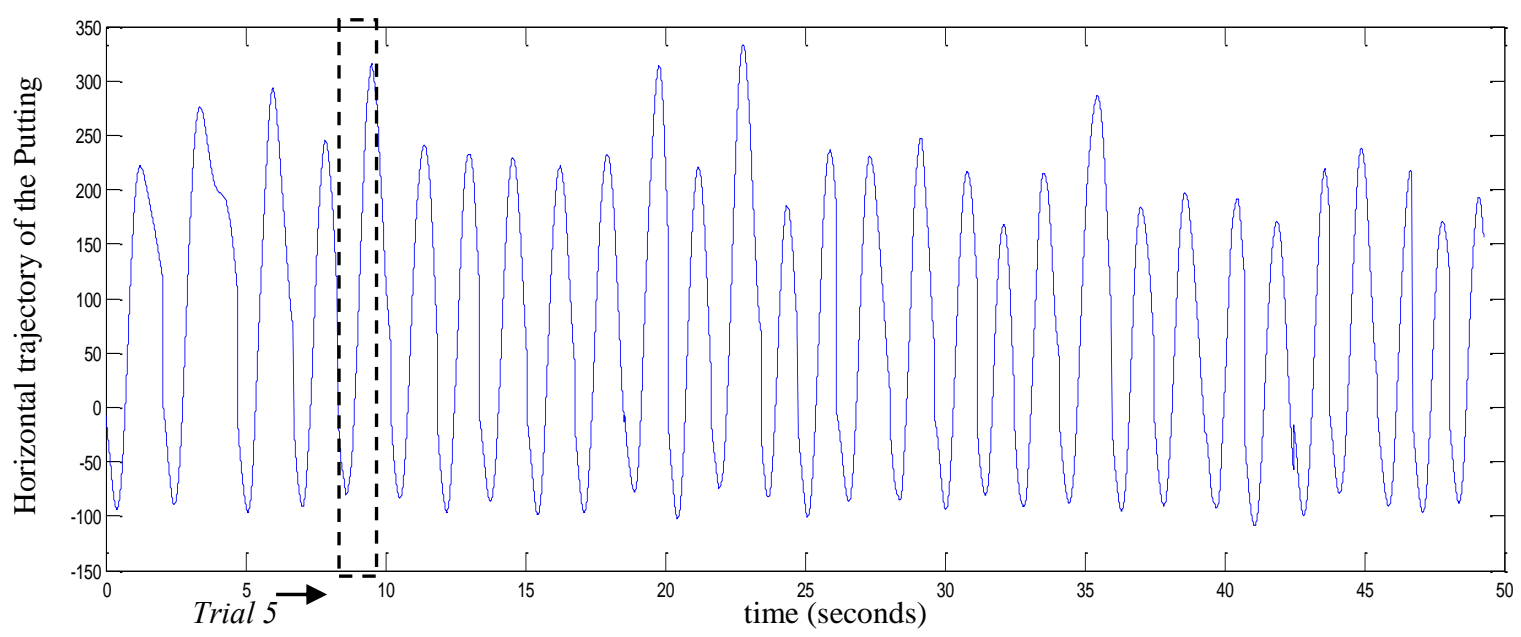

Figure 2. Example of the concatenation of 30 trials from a given golfer and practice condition.

Having a time-series, one can now directly apply the approximate entropy (ApEn) (section 2.2) to analyze the variability of golf putting over all practice conditions [9]. Table 1 depicts the approximate entropy for the motor execution of the putting of each golfer in the three studies. Highest ApEn values are highlighted to represent the practice conditions within each study that led to a higher human variability.

The results indicate that the golf putting performance can be described as a nonlinear, stable and regular system in which each golfer discovers active solutions to overcome the constraints of the task [9], [21], [40]. Interestingly, the ApEn values found throughout the three studies show that the minimum values are generally obtained at longer distances $(\mathbf{4 m})$, increasing almost linearly as the golfer gets near the hole. This is highly unexpected as it means that golfers depict a larger variability when near the hole. Not that unexpected was the result found for the third study, in which the maximum value of ApEn may be observed for the Angle Two (A2), when golfers had to apply a curvilinear trajectory in order for the ball to overcome the ramp. Note that this situation is considerably more complex than the alternatives, including Angle One (A1), as golfers have their vision partially constrained (see Figure 1a), thus being under a large amount of 'noise' and variability. In terms of the athletes, Players 1 and 9 proved to be the most consistent (presenting the lowest $A p E n$ ), whereas Players 3, 6 and 8 presented the highest levels of entropy (Figure 3). 
Table 1. Approximate entropy for motor execution of putting of each golfer in three studies.

\begin{tabular}{|c|c|c|c|c|c|c|c|c|c|c|c|c|}
\hline & PC & P1 & P2 & P3 & P4 & P5 & P6 & P7 & P8 & P9 & P10 & Avg per St \\
\hline \multirow{4}{*}{$\bar{E}$} & $1 \mathrm{~m}$ & 0.042 & 0.056 & 0.071 & 0.055 & 0.056 & 0.068 & 0.057 & 0.073 & 0.047 & 0.057 & 0.058 \\
\hline & $2 \mathrm{~m}$ & 0.042 & 0.056 & 0.068 & 0.053 & 0.052 & 0.071 & 0.048 & 0.065 & 0.044 & 0.051 & 0.055 \\
\hline & $3 \mathrm{~m}$ & 0.046 & 0.053 & 0.068 & 0.043 & 0.043 & 0.055 & 0.047 & 0.061 & 0.041 & 0.046 & 0.050 \\
\hline & $4 \mathrm{~m}$ & 0.044 & 0.045 & 0.060 & 0.037 & 0.041 & 0.052 & 0.056 & 0.056 & 0.043 & 0.049 & 0.048 \\
\hline \multirow{3}{*}{ कै } & $2 \mathrm{~m}$ & 0.040 & 0.053 & 0.062 & 0.062 & 0.040 & 0.058 & 0.051 & 0.069 & 0.043 & 0.065 & 0.0543 \\
\hline & $3 \mathrm{~m}$ & 0.033 & 0.048 & 0.064 & 0.054 & 0.039 & 0.066 & 0.064 & 0.058 & 0.044 & 0.048 & 0.0518 \\
\hline & $4 \mathrm{~m}$ & 0.036 & 0.041 & 0.064 & 0.044 & 0.040 & 0.051 & 0.049 & 0.054 & 0.036 & 0.046 & 0.0461 \\
\hline \multirow{2}{*}{$\ddot{m}$} & $\mathrm{~A} 1$ & 0.040 & 0.055 & 0.056 & 0.051 & 0.042 & 0.059 & 0.053 & 0.075 & 0.054 & 0.055 & 0.054 \\
\hline & $\mathrm{A} 2$ & 0.050 & 0.066 & 0.054 & 0.061 & 0.065 & 0.083 & 0.070 & 0.076 & 0.053 & 0.056 & 0.063 \\
\hline
\end{tabular}

Figure 3 complements the aforementioned table by presenting the average entropy for each golfer over all practice conditions.

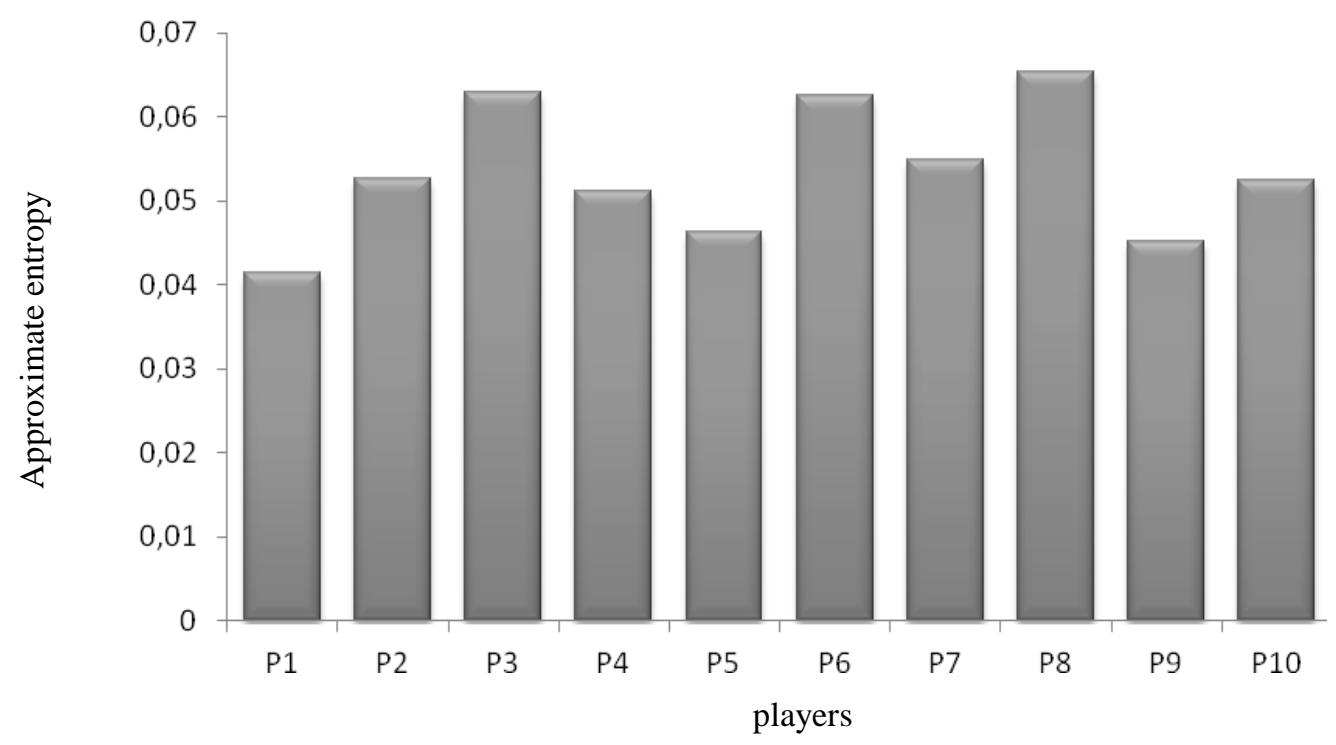

Figure 3: Average approximate entropy for each golfer.

Out of curiosity, when calculating the overall average $A p E n$, the putting performance of expert golfers yields a value of 0.053 . This is a very stable, regular and periodic value [9]. As one will observe, the next case study does not share the same regularity.

\subsection{Tennis}

In this section, let us now increase the complexity of the human motor execution from a pendulum to a ballistic-like motion. For the tennis serve case, a non-traditional analysis of motor variability may help revealing or uncovering its complex structure, which is undetectable using conventional linear measures, such as amplitude or standard deviation [28], [29]. In this case study, we aimed at studying the intra and 
inter-individual variability of motor performance in the first serve using the approximate entropy, while manipulating the lateral wind constraint by benefiting from an induced aerodynamic flow (IAF) device which was adapted from an industrial helical fan METEC-HCT-45-4T (Figure 4). Please, refer to [30] for a more detailed description of the setup.

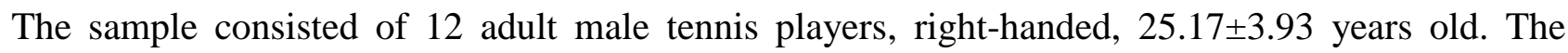
anthropometric characteristics of this group were as follows: height $177 \pm 6.00 \mathrm{~cm}$, wingspan of $181 \pm 5.00$ $\mathrm{cm}$ and body mass of $72.29 \pm 4.17 \mathrm{Kg}$. All athletes had been playing tennis for $16.25 \pm 5.56$ years, from which $13.67 \pm 4.29$ years were at the national competition level. The indoor tennis court had the official dimensions for the singles tennis game, with $2377 \mathrm{~cm}$ long and $823 \mathrm{~cm}$ wide.

The movement under study was the flat first serve from behind the base line of the tennis court, on the right-hand side, and $80 \mathrm{~cm}$ away from the center mark. All the participants were asked to serve at a maximum speed and aiming at the point of intersection between the center line and the service line (i.e., "T-point"). Also, they were all asked to perform 20 tennis serves, without instructional or wind constraints, called IAF0 (a control condition). Afterwards, they were then asked to perform four sets of 20 serves under different practice conditions: (1) minimum IAF speed of $2.4 \mathrm{~m} . \mathrm{s}^{-1}$ (called IAF1); (2) medium IAF speed of $4.3 \mathrm{~m} . \mathrm{s}^{-1}$ (called IAF2), 3); (3) maximum IAF speed of $5.8 \mathrm{~m} . \mathrm{s}^{-1}$ (called IAF3) and; (4) random IAF speed with random sequences at the three IAF speeds (called IAFr). All these then accounts for 100 serves for each athlete.

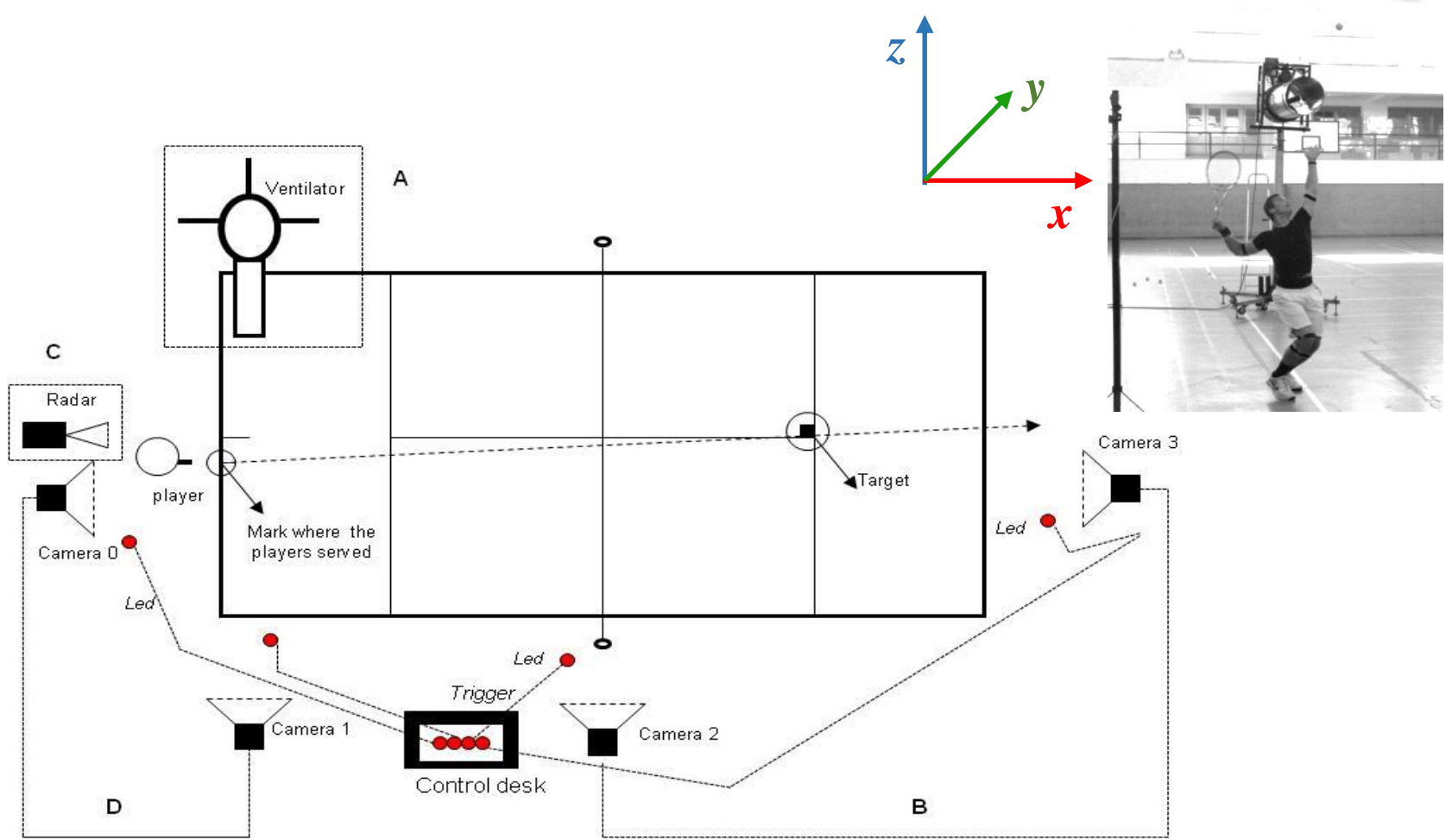

Figure 4. Experimental set up. A - Device IAF. B - Accuracy registration device. C - Device for recording the velocity of the serve. D - Device for analyzing the impact point in the serve. Dashed arrow indicates a possible direction of tennis serve: adapted from [30]. 
The recording of the racket motion was obtained from two cameras operating at $210 \mathrm{~Hz}$ (cameras 0 and 1 from Figure 4). The cameras were synchronized using a common light-emitting diode (LED) as a visual trigger [30]. A 3D analysis of the collected images was carried out. For a more detailed description of the 3D analysis, please refer to [31].

Table 2. Approximate entropy for motor execution of tennis serve of each player in five practical conditions, in three components $x, y$ e $z$.

\begin{tabular}{|c|c|c|c|c|c|c|}
\hline \multirow{2}{*}{\multicolumn{2}{|c|}{ Participants }} & \multicolumn{5}{|c|}{ Practical Conditions } \\
\hline & & IAFO & IAF1 & IAF2 & IAF3 & IAFr \\
\hline \multirow{3}{*}{1} & $A p E n \_x$ & 0.0347 & 0.0309 & 0.0307 & 0.0307 & 0.0324 \\
\hline & $A p E n \_y$ & 0.0534 & 0.0506 & 0.0562 & 0.0045 & 0.0525 \\
\hline & $A p E n \_z$ & 0.1070 & 0.0926 & 0.0892 & 0.0040 & 0.0954 \\
\hline \multirow{3}{*}{2} & $A p E n \_x$ & 0.0171 & 0.0188 & 0.0272 & 0.0315 & 0.0363 \\
\hline & $A p E n \_y$ & 0.0374 & 0.0375 & 0.0414 & 0.0413 & 0.0386 \\
\hline & $A p E n \_z$ & 0.0209 & 0.0196 & 0.0202 & 0.0204 & 0.0197 \\
\hline \multirow{3}{*}{3} & $A p E n \_x$ & 0.0213 & 0.0259 & 0.0279 & 0.0252 & 0.0238 \\
\hline & $A p E n \_y$ & 0.0222 & 0.0235 & 0.0250 & 0.0248 & 0.0227 \\
\hline & $A p E n \_z$ & 0.0330 & 0.0321 & 0.0341 & 0.0305 & 0.0287 \\
\hline \multirow{3}{*}{4} & $A p E n \_x$ & 0.0171 & 0.0125 & 0.0326 & 0.0350 & 0.0347 \\
\hline & $A p E n \_y$ & 0.0007 & 0.0411 & 0.0347 & 0.0410 & 0.0320 \\
\hline & $A p E n \_z$ & 0.0209 & 0.0374 & 0.0223 & 0.0310 & 0.0212 \\
\hline \multirow{3}{*}{5} & $A p E n \_x$ & 0.0316 & 0.0348 & 0.0344 & 0.0331 & 0.0392 \\
\hline & $A p E n \_y$ & 0.0351 & 0.0330 & 0.0374 & 0.0383 & 0.0447 \\
\hline & $A p E n \_z$ & 0.0374 & 0.0397 & 0.0432 & 0.0412 & 0.0353 \\
\hline \multirow{3}{*}{6} & $A p E n \_x$ & 0.0559 & 0.0265 & 0.0261 & 0.0276 & 0.0310 \\
\hline & $A p E n \_y$ & 0.0278 & 0.0410 & 0.0425 & 0.0355 & 0.0223 \\
\hline & $A p E n \_z$ & 0.0230 & 0.0354 & 0.0233 & 0.0377 & 0.0282 \\
\hline \multirow{3}{*}{7} & $A p E n \_x$ & 0.0289 & 0.0311 & 0.0322 & 0.0278 & 0.0301 \\
\hline & $A p E n \_y$ & 0.0298 & 0.0245 & 0.0236 & 0.0280 & 0.0259 \\
\hline & $A p E n \_z$ & 0.0228 & 0.0222 & 0.0219 & 0.0214 & 0.0215 \\
\hline \multirow{3}{*}{8} & $A p E n \_x$ & 0.0171 & 0.0267 & 0.0436 & 0.0290 & 0.0313 \\
\hline & $A p E n \_y$ & 0.0366 & 0.0312 & 0.0467 & 0.0334 & 0.0340 \\
\hline & $A p E n \_z$ & 0.0308 & 0.0318 & 0.0290 & 0.0346 & 0.0353 \\
\hline \multirow{3}{*}{9} & $A p E n \_x$ & 0.0354 & 0.0386 & 0.0345 & 0.0386 & 0.0356 \\
\hline & $A p E n \_y$ & 0.0195 & 0.0230 & 0.0218 & 0.0242 & 0.0220 \\
\hline & $A p E n \_z$ & 0.0218 & 0.0255 & 0.0223 & 0.0249 & 0.0261 \\
\hline \multirow{3}{*}{10} & $A p E n \_x$ & 0.0272 & 0.0331 & 0.0329 & 0.0334 & 0.0271 \\
\hline & $A p E n \_y$ & 0.0279 & 0.0299 & 0.0310 & 0.0334 & 0.0336 \\
\hline & $A p E n \_z$ & 0.0300 & 0.0251 & 0.0293 & 0.0241 & 0.0201 \\
\hline \multirow{3}{*}{11} & $A p E n \_x$ & 0.0168 & 0.0315 & 0.0271 & 0.0293 & 0.0262 \\
\hline & $A p E n \_y$ & 0.0409 & 0.0523 & 0.0520 & 0.0477 & 0.0490 \\
\hline & $A p E n \_z$ & 0.0225 & 0.0287 & 0.0309 & 0.0351 & 0.0335 \\
\hline \multirow{3}{*}{12} & $A p E n \_x$ & 0.0329 & 0.0402 & 0.0372 & 0.0454 & 0.0412 \\
\hline & $A p E n \_y$ & 0.0245 & 0.0236 & 0.0240 & 0.0231 & 0.0256 \\
\hline & $A p E n \_z$ & 0.0263 & 0.0255 & 0.0265 & 0.0243 & 0.0229 \\
\hline $\begin{array}{c}\text { Total } \\
\text { ApEn_x }\end{array}$ & Avg per $S t$ & 0.0303 & 0.0307 & 0.0322 & 0.0307 & 0.0302 \\
\hline $\begin{array}{c}\text { Total } \\
\text { ApEn_y }\end{array}$ & $A v g$ per $S t$ & 0.0292 & 0.0343 & 0.0343 & 0.0314 & 0.0337 \\
\hline $\begin{array}{c}\text { Total } \\
\text { ApEn_z }\end{array}$ & $A v g$ per $S t$ & 0.0335 & 0.0346 & 0.0344 & 0.0271 & 0.0322 \\
\hline
\end{tabular}


The approximate entropy $(A p E n)$ was calculated based on the position of the racket during the execution of the service, considering the three components $x, y$ and $z$-axis. Table 2 depicts the approximate entropy for the motor execution of the tennis serve of each player in the five practice conditions. Highest $A p E n$ values are highlighted to represent the practice conditions that led to a higher human variability in each axis.

The $A p E n \_x$ in the lateral component ( $x$-axis) has shown that all participants present values inferior to 0.0560 . In general, IAF2 was the condition that yielded higher variability in the $x$-axis. Regarding the depth component ( $y$-axis), ApEn_y values were similarly low (less than 0.0562). Again, IAF2, now together with IAF1, were the conditions that yielded higher variability, this time in the $y$-axis. In the vertical component, $A p E n \_z$ values were even lower, below 0.0432. Player 1 presented an overall larger variability in most conditions but, in general, the variability in the vertical component was rather smaller than expected. Still, IAF1 was the condition that yielded higher variability in the $z$-axis.

This nonlinear analysis carried out for the racquet position along the three axes resulted in an approximate entropy close to 0 (inferior to 0.1 ).To some extent, this characterizes the serve like a periodic system with high regularity and low variability, regardless on the wind conditions [15]. Although the study of Menayo [32] reached the same conclusions using different methods for players of intermediate level under different constraints, we were expecting to observe a more chaotic behavior from a ballistic movement. It is also noted that, even though it may be neglected in some occasions, there has been a larger number of players with a slight tendency to increase their variability due to wind constraints.

\subsection{Soccer}

As one may observe from the previous case studies, the approximate entropy can be applied to study athletes' variability in different contexts. In this section, we consider both entropy-based measures, namely Shannon's entropy and the approximate entropy to analyze the variability inherent to soccer players' trajectories.

In a first instance, let us consider the applicability of Shannon's entropy. According to section 2.1, to do so one should first divide the soccer field into cells. To do so, one can segment the field into $1 \mathrm{~m}^{2}$ resolution. The time a given player stands in each $1 \mathrm{~m}^{2}$ cell will result into a specific heat map (i.e., histograms) representing the overall trajectory travelled by him inside the soccer field [33], [34]. For the sake of simplicity, the player's position has been discretized at each second, in which a given cell gets the value of 1 to identify the player presence, or 0 otherwise [35]. This spatio-temporal approach allows to identify the spatial variability of a soccer player trajectory over the whole match. Figure $\mathbf{5}$ presents a specific case of how the variability can be understood by considering different tactical positions. 


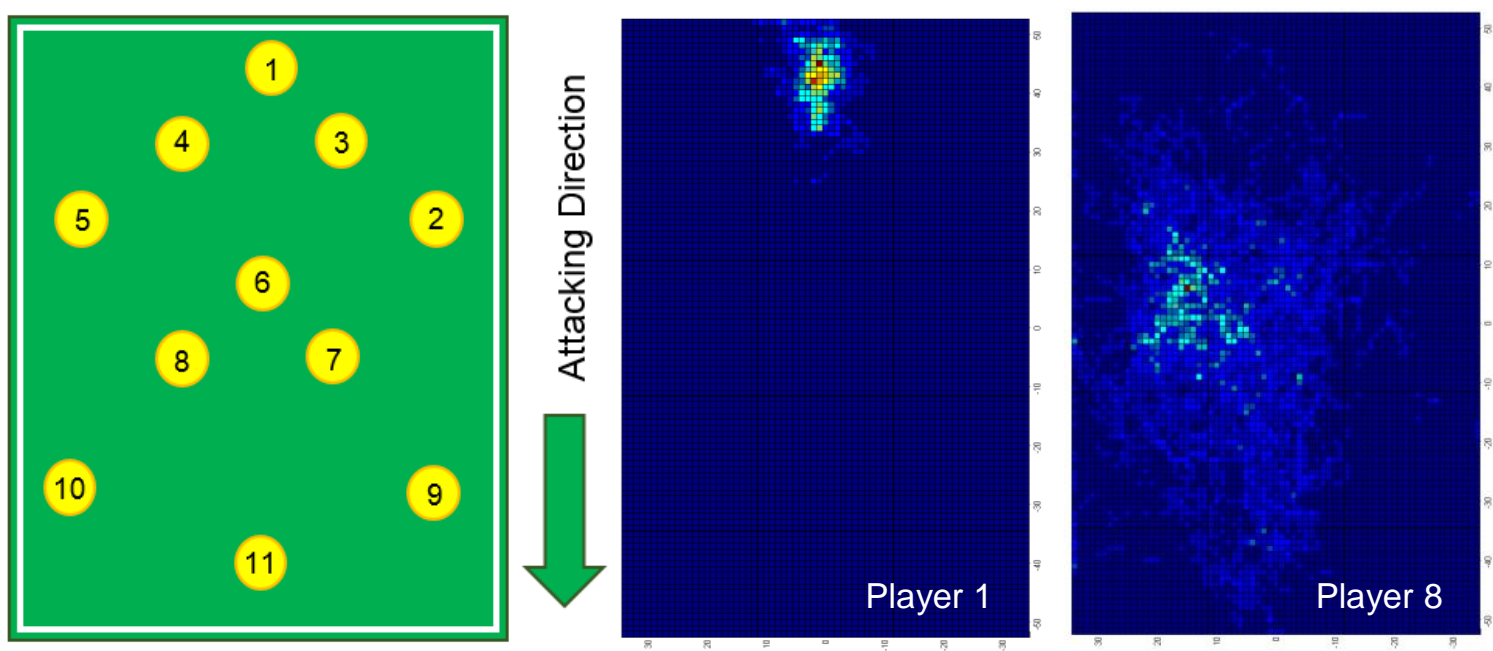

Figure 5. a) Tactical line-up of team; b) Goalkeeper's heat map (Player 1); c) Midfielder's heat map (Player 8).

As one can observe in Figure 5b,c, the soccer players' trajectories vary accordingly to their tactical position, being defined by the strategic plan and by the game dynamics [36]. It is noteworthy that Figure $\mathbf{5 b , c}$ is a typical representation of an image with three dimensions and, henceforth, one can apply Shannon's entropy as it would be the case of any other histogram. In this work, we considered an entire soccer match and computed Shannon's entropy for all soccer players [17]. Figure 6 depicts the results.

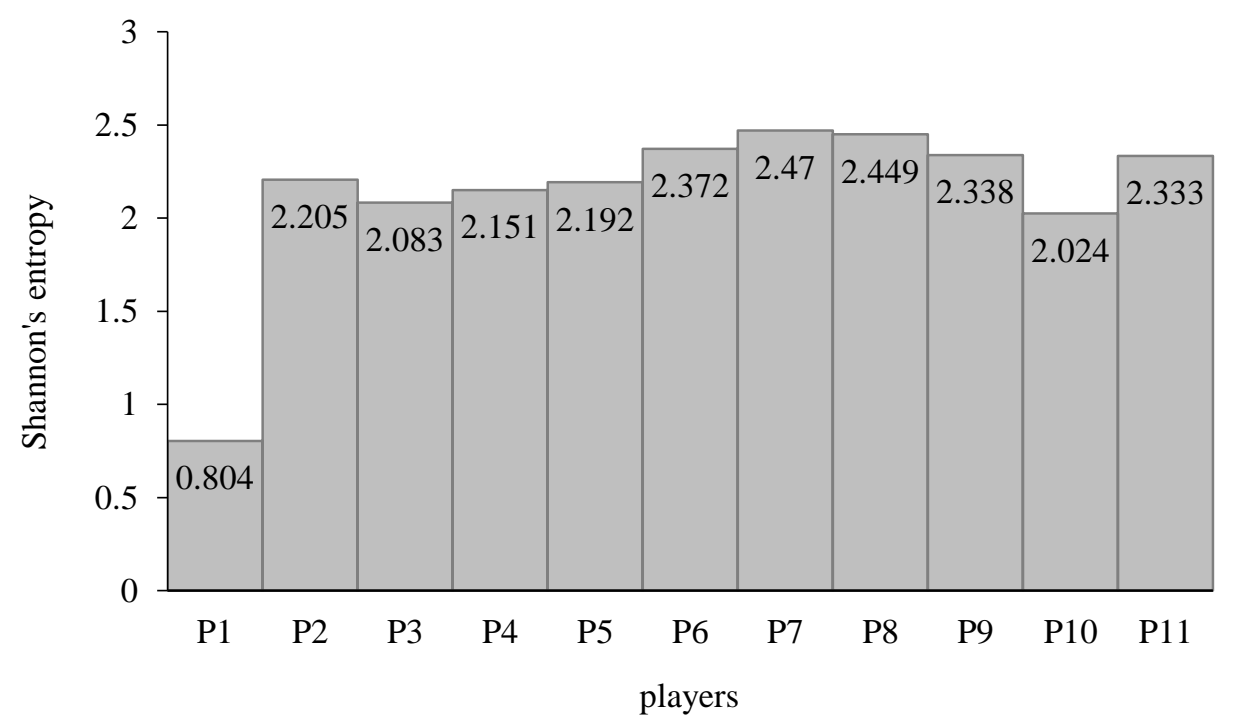

Figure 6. Shannon's entropy of all players from one of the teams during an entire soccer match.

In this case study, it was found that midfielders present a larger Shannon's entropy (player 7: $E=$ 2.470; player 8: $E=2.449$; player 6: $E=2.372$ ). As expected, the lowest value was found for the goalkeeper $(E=0.804)$. These results reveal that midfielders cover a larger area and spend less time in a specific region of the field. This can be explained by their tactical role, mainly because they have an active participation linking both defensive and offensive sectors [37]. 
Nevertheless, Shannon's entropy considers the overall of players without making an allowance for the sequence generating such a trajectory, i.e., the sequence of cells a given player was in. Although Shannon's entropy can be used for coaches and their staff to control the physical demands of a match and to assess players' performance over the time [38], this kind of analysis completely excludes the temporal factor. However, it is quite important to understand how players react during the match in order to identify possible motion patterns. This can be an invaluable information to optimize daily training sessions by exploiting the cycles of low and high intensities emerging during the match.

To better illustrate the previous argument, let us show an example of the distance covered by a player over time (Figure 7).

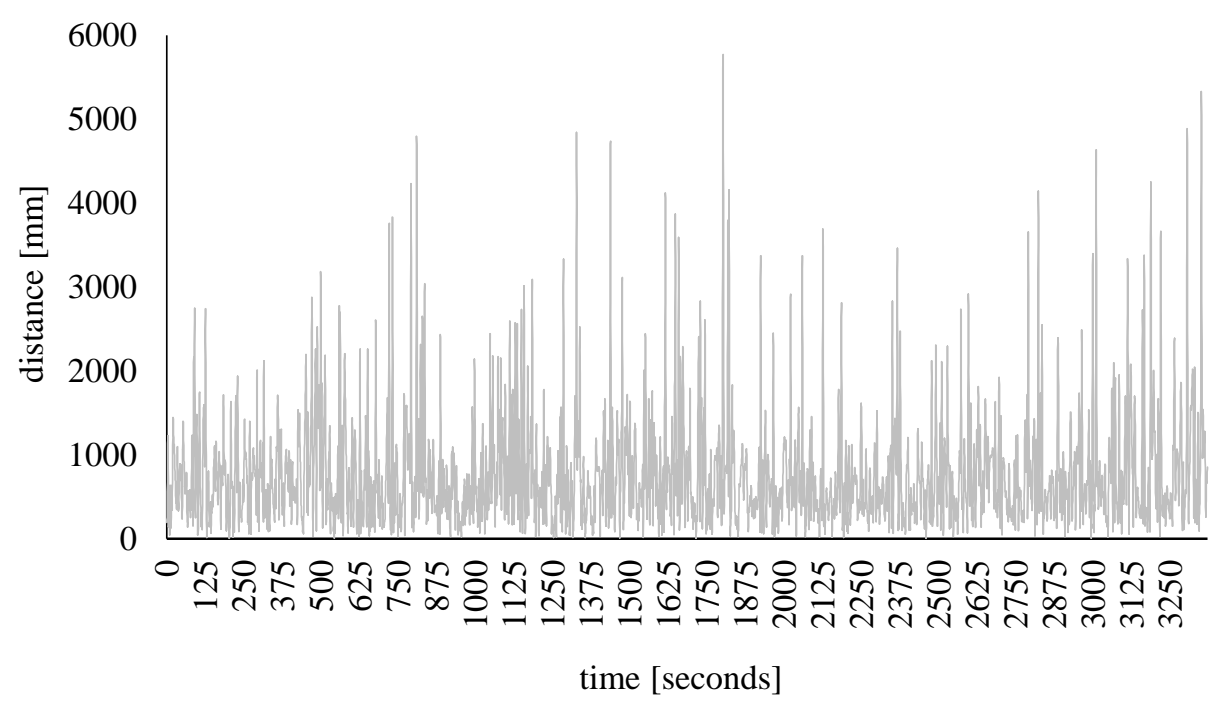

Figure 7. Distance covered by a player during an entire soccer match.

The example depicted in Figure 7 shows that players do not cover the same distance at each second. Hence, the variability inherent to a given player's trajectory is not constant and must be managed by himself and by the coach, in order to anticipate and avoid the human fatigue that may affect his performance [39]. As previously stated, the variability depends from the tactical role and even from the strategic plan defined by the coach to manage the physiological responses of players.

For this case, and following a similar approach from the one adopted for the previous case studies, the approximate entropy was considered [17]. Figure 8 depicts the obtained results. 


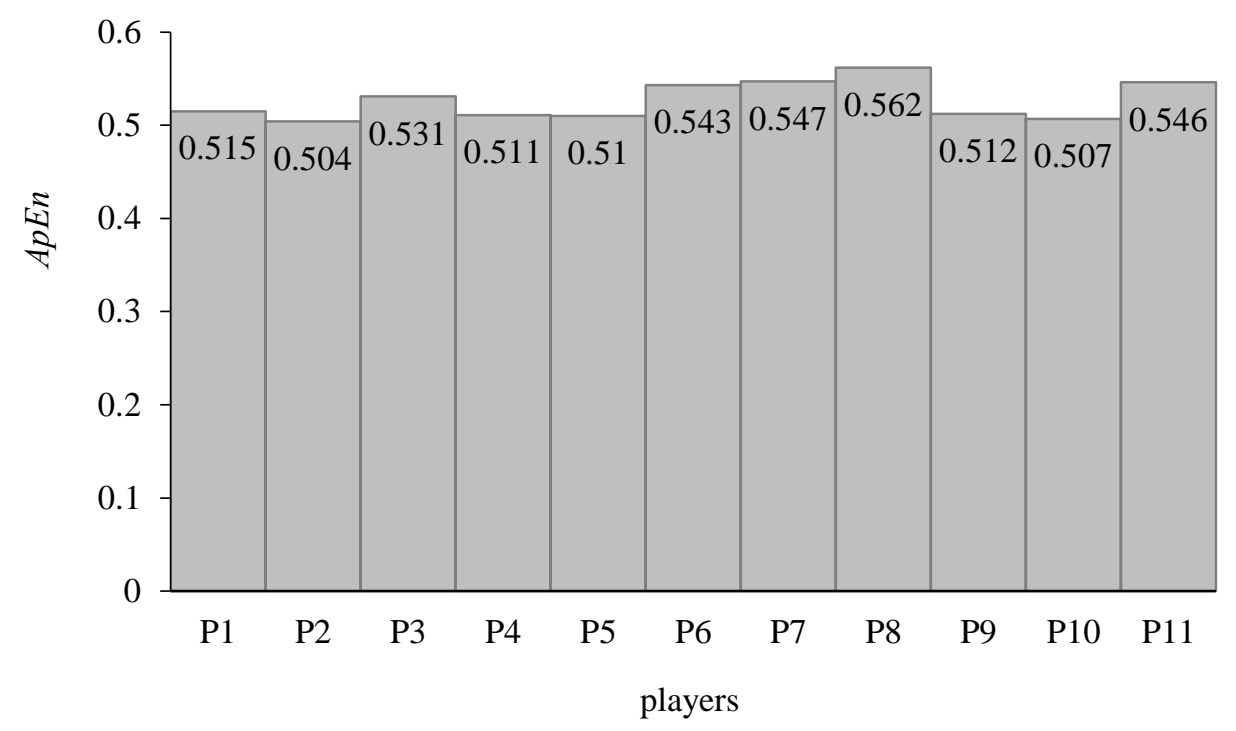

Figure 8. Approximate entropy of all players from one of the teams during an entire soccer match.

As opposed to Shannon's entropy, the approximate entropy did not showed any major differences between players' variability. Yet, the largest values were found for two of the midfielders (player 7: 0.547; and player 8: 0.562) and one forward player (player 11: 0.546). These values, similarly as observed before, are inherent to players presenting a larger mobility during the match. Once again, the distance travelled seems to be directly related with the variability.

\section{Conclusions}

From the case studies considered in this paper, one can concluded that nonlinear techniques are extremely useful to analyze the human movement within sports context. In general, entropy-based measures can be considered as one of the main methods to study the human variability, thus making up for the limitations inherent to linear techniques typically used to quantify the motor performance, such as the standard deviation, the inter-quartile range, and many other classical alternatives. However, it is noteworthy that we do not aim at underestimating the role that linear techniques have in sports sciences, but rather propose alternative nonlinear methods that may deepen our understanding around the human movement science.

In this paper, both Shannon's entropy and approximate entropy are considered to further understand human variability in the context of the golf putting (pendulum-like movement), tennis serve (ballisticlike movement) and football players' trajectories (chaotic/random movement). The insights provided in this work bring important practical implications to the area of sports training, since these suggest that the athlete can stabilize his performance by exploiting different configurations of information-movement within different levels of variability, regularity and complexity. This reveals the complexity inherent in normal variability, indicating motor control features that are imperative for athletes and coaches to measure and, more importantly, to understand. Moreover, the application of principles based on nonlinear dynamics can provide a novel perspective on how training in general should be organized.

At last, it should be noted that athletes' characteristics (morphological and functional), their level of performance and the complexity inherent in motor execution play an important role in the outcome 
provided by the entropy measures. Consequently, it is the authors' belief that the solution to the problem of individuality should not be limited to the use of ideal or standardized techniques, but yet it shall contemplate a wide variety of nonlinear strategies that can be implemented and adapted to the specificity of each athlete.

\section{Conflicts of Interest}

The authors declare no conflict of interest.

\section{References and Notes}

1. Kudo, K.; Ohtsuki T. Adaptive variability in skilled human movements. Information and media technologies 2008, 3, 409-420.

2. Araújo, D.; Davids, K.; Bennett, S.; Button, C.; Chapman, G. Emergence of sport skills under constraint. In Skill Acquisition in Sport: Research, Theory and Practice. Williams, A.; Hodges, N.; Eds.; Routledge, Taylor \& Francis, London, 2004, pp. 409-433.

3. Bernstein, N.A. The co-ordination and regulation of movements; Pergamon Press: New York, USA, 1967.

4. Tözeren A. Human Body Dynamics: Classical Mechanics and Human Movement; Springer-Verlag New York, USA, 2000.

5. Newell, K.M., James, E.G. The amount and structure of human movement variability. Routledge Handbook of Biomechanics and Human Movement Science; Taylor \& Francis New York, USA, Group, 2008.

6. Diener, H.C.; Bootz, F., Dichgans, J.; Bruzek, W. Variability of postural "reflexes" in humans. Experimental Brain Research 1983, 52, 423-428.

7. Newell, K.M.; Vaillancourt, D.E. Dimensional change in motor learning. Human Movement Science 2001, 20, 695-715.

8. Couceiro, M.S.; Dias, G.; Mendes, R.; Araújo, D. Accuracy of pattern detection methods in the performance of golf putting. Journal of Motor Behaviour 2013, 45, 37-53.

9. Dias, G.; Couceiro, M.S.; Clemente, M.S., Martins, F.M., \& Mendes, R.. A non-linear understanding of golf putting. SA Journal for Research in Sport, Physical Education and Recreation 2014, 36, 61-77.

10. Dias, G.; Couceiro, M.S.; Barreiros, J.; Clemente, M.S.; Mendes, R.; Martins, F.M. Distance and slope constraints: Adaptation and variability in golf putting. Motor Control 2014, 18, 221-243.

11. Stergiou, N.; Yu, Y.; Kyvelidou, A. (2013). A Perspective on Human Movement Variability with Applications in Infancy Motor Development. Kinesiology Review 2013, 2, 93-102. 
12. Stergiou, N.; Buzzi, U.H.; Kurz, M.J.; Heidel, J. (2004). Non-linear tools in human movement. In Innovative analyses of human movement. Stergiou, N.; Eds.; Champaign, Human Kinetics, Illinois, :2004, pp. 163-186.

13. Adams, J.A. (1971). A closed-loop theory of motor learning. Journal of Motor Behaviour 1971, 3 , 111-150.

14. Schmidt, R.A. A schema theory of discrete motor skill learning. Psychological Review 1975, 82, 225-260.

15. Harbourne, R.T.; Stergiou, N. Movement variability and the use of non-linear tools: Principles to guide physical therapist practice. Journal of Neurologic Physical Therapy 2009, 3, 267-282.

16. Sampaio J.; Maçãs, V. Measuring tactical behaviour in football. International Journal of Sports Medicine 2012, 33, 395-440.

17. Couceiro, M.S.; Clemente, F.M.; Martins, F.M.L.; Tenreiro Machado, J.A. Dynamical Stability and Predictability of Football Players: The Study of One Match. Entropy 2014, 16, 645-674.

18. Shannon, C.E. A Mathematical Theory of Communication. The Bell System Technical Journal 1948, 27, 623-656.

19. Silva, M.L.; Piqueira, J.R.C.; Vielliards, J.M.E. Using Shannon Entropy on Measuring the Individual Variability in the Rufous-bellied Thrush Turdus rufiventris Vocal Communication. Journal of theoretical biology 2000, 207, 57-64.

20. Kurths, J.; Voss, A.; Saparin, P.; Witt, A.; Kleiner, H.J.; Wessel N. Quantitative analysis of heart rate variability. Chaos: An Interdisciplinary Journal of Nonlinear Science 1995, 5, 88-94.

21. Couceiro, M.S.; Dias, G.; Martins, F.M.L.; Luz, J.M. A fractional calculus approach for the evaluation of the golf lip-out. Signal, Image and Video Processing 2012, 6, 437-443.

22. Sabuncu, M.R. Entropy-based Image Registration. PhD Thesis: Faculty of Princeton University, Candidacy, 2006.

23. Allen, D.E.; McAleer, M.; Singh, A.J. An entropy based analysis of the relationship between the DOW JONES Index and the TRNA Sentiment series. QUANTVALLEY/FdR: "Quantitative Management Initiative”, 2013. Retrieved from www.qminitiative.org/quantitativemanagementinitiative.html.

24. Pincus, S.M. Approximate entropy as a measure of system complexity. Proceedings of the National Academy of Sciences, USA 1991, 88, 2297-2301. 
25. Balasis, G.; Donner, R.V.; Potirakis, S.M.; Runge, J., Papadimitriou, C.; Daglis, I.A.; Eftaxias, K.; Kurths, J. Statistical mechanics and information-theoretic perspectives on complexity in the earth system. Entropy 2013, 15, 4844-4888.

26. Grassberger, P., Procaccia, I. Estimation of the Kolmogorov entropy from a chaotic signal. Physical Review A 1983, 28, 2591-2593.

27. Fonseca, S.; Milho, J.; Passos, P.; Araújo, D.; Davids, K. Approximate entropy normalized measures for analyzing social neurobiological systems. Journal of motor behavior 2012, 44, 179-83.

28. Liebovitch, L.S.; Todorov, A.T. Invited editorial on "Fractal dynamics of human gait: Stability of long-range correlations in stride interval fluctuations." Journal of Applied Physiology 1996, 80, 14461447.

29. Post, A.A.; Daffertshofer, A.; Beek, P.J. Principal components analysis in three-ball cascade juggling. Biological Cybernetics 2000, 82, 143-152.

30. Mendes, P.C.; Dias, G.; Mendes, R.; Martins, F.M.L.; Couceiro, M.S.; Araújo, D. The effect of artificial side wind on the serve of competitive tennis players. International Journal of Performance Analysis in Sport 2012, 12, 546-562.

31. Mendes, P.C.; Fuentes, J.P.; Mendes, R.; Martins, F.M.L.; Clemente, F.; Couceiro, M.S. The variability of the serve toss in tennis under the influence of artificial crosswind. Journal of Sports Science and Medicine 2013, 12, 309-315.

32. Menayo, R. Análisis de la relación entre la consistencia en la ejecución del patrón motor del servicio en tenis, la precisión y su aprendizage en condiciones de variabilidad. Dissertação (Doutoramento em Ciencias del Deporte) - Facutad de Ciencias del Desporte, Universidad de Extremadura, Cáceres 2010.

33. Martins, F.M.L.; Clemente, F.M.; Couceiro, M.S. From the individual to the collective analysis at the football game. Proceedings on Mathematical Methods in Engineering International Conference, Porto, Portugal 2013.

34. Clemente, F.M.; Couceiro, M.S.; Martins, F.M.; Dias, G., Mendes, R. Interpersonal dynamics: 1v1 sub-phase at sub-18 football players. Journal of Human Kinetics 2013, 36, 179-89.

35. Duque C. Priors for the Ball Position in Football: Match using Contextual Information. Stockholm, Sweden Royal Institute of Technology, School of Computer Science and Communication, 2010.

36. Clemente, F.M.; Couceiro, M.S.; Martins, F.M., Mendes, R., Figueiredo, A.J. Measuring tactical behaviour using technological metrics: Case study of a football game. International Journal of Sports Science \& Coaching 2013, 8, 723-39. 
37. Bloomfield, J.; Polman, R., O'Donoghue, P. Physical demands of different positions in FA Premier League soccer. Journal of sports science \& medicine 2007, 6, 233-242.

38. Carling, C.; Bloomfield, J.; Nelsen, L.; Reilly, T. The role of motion analysis in elite soccer. Sports Medicine 2008, 38, 839-862.

39. Bangsbo J. The physiology of soccer - with special reference to intense intermittent exercise. Acta physiologica Scandinavica 1994, 619, Supplementum 02, 1-155.

40. Pelz, D. Putting bible: The complete guide to mastering the green. Doubleday: New York, 2000.

(C) 2014 by the authors; licensee MDPI, Basel, Switzerland. This article is an open access article distributed under the terms and conditions of the Creative Commons Attribution license (http://creativecommons.org/licenses/by/3.0/). 